\title{
sensors
}

ISSN 1424-8220

(C) 2001 by MDPI

http://www.mdpi.net/sensors

\section{Magnetism-Based Remote Query Glucose Sensors}

\author{
K. G. Ong, M. Paulose, M. K. Jain, D. Gong, O. K. Varghese, C. Mungle and C. A. Grimes* \\ Department of Electrical Engineering and Materials Research Institute, The Pennsylvania State \\ University, 208 Materials Research Lab, University Park, PA 16802.
}

* Author to whom correspondence should be addressed. E-mail: cgrimes@engr.psu.edu

Received: 18 September 2001 / Accepted: 28 September 2001 / Published: 7 October 2001

\begin{abstract}
Two wireless, passive remote query magnetism-based glucose sensors, which operate in combination with a mass and volume changing glucose responsive polymer, are presented. One sensor design is based upon the magnetostatic coupling of magnetically soft thin-film elements; as the polymer volume changes in response to glucose concentration so does the magnetostatic coupling between elements. In response to a time varying magnetic field, upon reversal of the magnetization vector of the elements the magnetostatic coupling determines the time rate of change of magnetic flux, and hence the amplitude of the voltage spike generated in a pick-up coil. The other sensor consists of a free-standing magnetoelastic thick-film, coated with a thin layer of the glucose responsive polymer. In response to a time varying magnetic field the sensor mechanically vibrates at a characteristic resonant frequency; the characteristic resonant frequency of the sensor linearly tracks the change in mass of the glucose responsive polymer.
\end{abstract}

Keywords: Glucose sensor, Magnetostatic coupling, Magnetoelastic, Magnetostrictive, remote query.

\section{Introduction}

Sensors for blood glucose concentration are of great medical and commercial interest. Most glucose sensors are based upon the amperometric response of electrodes coated with glucose responsive materials; as the glucose concentration changes so does the electrical properties of the responsive materials, and hence the electrical current measured across the electrodes [1-4]. A limitation of these sensors is that they require direct wire connections between the electrodes and the detection 
electronics, which generally precludes their use in-vivo. In contrast to electronic devices requiring direct connections, magnetism-based devices operate through monitoring changes in magnetic flux using a remotely located pick-up coil. The remote nature of magnetism-based devices offers exciting opportunities for both in-situ and in-vivo monitoring.

In this paper we present two wireless, passive magnetism-based glucose sensors that operate in combination with a mass and volume changing glucose responsive polymer. One sensor consists of magnetically soft (low coercive force, high permeability) thin-film elements adhered to a thick film of the glucose responsive polymer. The distance between the magnetic elements changes as the polymer swells and shrinks in response to changes in glucose concentration, changing the magnetostatic coupling between elements. This change in magnetostatic coupling between elements in turn modulates the coercive force and BH loop switching characteristics of the sensor. The other sensor is based on the mass-sensitive change in the magnetoelastic resonant frequency of an amorphous ferromagnetic glass ribbon coated with the glucose responsive polymer. In a manner analogous to mechanical resonators, for small mass loads, increasing mass linearly shift the resonant frequency of the magnetoelastic sensor lower. Both sensors convey information through magnetic flux, hence no electrical wire connections nor specific alignments are required. In addition, both sensors are powered by externally applied magnetic fields, so there are no power lifetime issues such as those associated with battery drain.

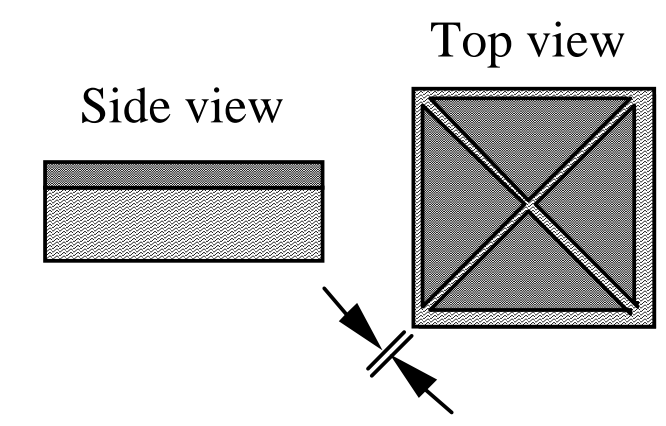

Soft magnetic material $23 \mu \mathrm{m}$ thick Glucose polymer $80 \mu \mathrm{m}$ thick

(a)

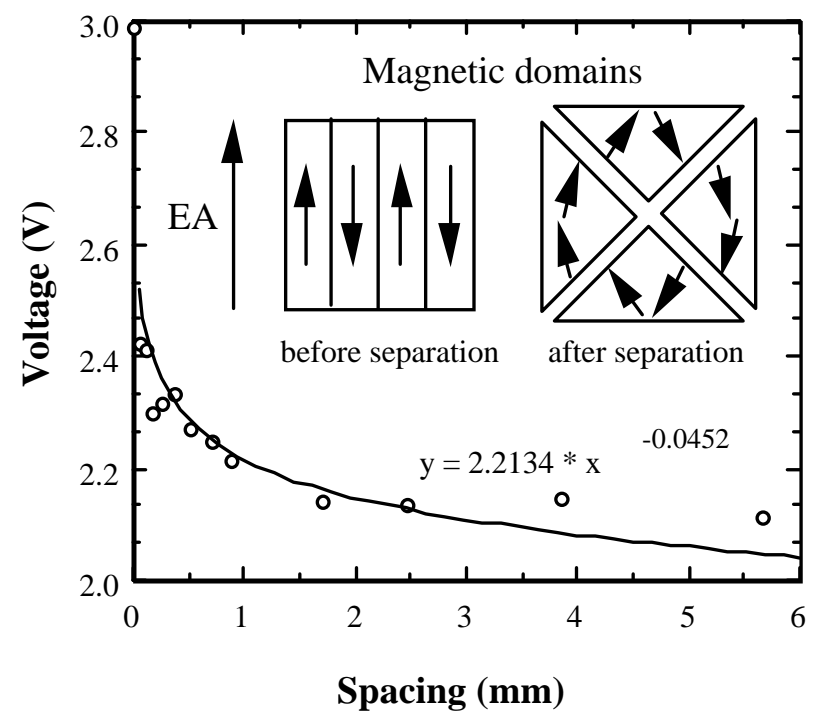

(b)

Figure 1. (a) Schematic drawing of the magnetostatic coupling glucose sensor. Initial spacing between elements is $50 \mu \mathrm{m}$, distance expands upon wetting and exposure to glucose. (b) Measured voltage spike amplitude vs. element spacing as a function of measured distance across diagonal. Domain drawing is schematic.

The magnetostatic coupling based sensor is shown in Fig. 1a. The sensor is composed of four triangular shaped, $23 \mu \mathrm{m}$-thick magnetically soft Atalante ${ }^{\circledR}$ films [5] bonded onto a physically robust $20 \mu \mathrm{m}$ thick film of a glucose responsive polymer (to be described) that swells/shrinks in response to changing glucose concentration. The triangles are spaced $50 \mu \mathrm{m}$ apart and arranged to form a $12 \mathrm{~mm} \times 12 \mathrm{~mm}$ square with the triangle tips centered at a common origin. When placed in an ac 
magnetic field of a magnitude sufficient to saturate the sample, $\approx 0.5 \mathrm{Oe}$, the time varying flux associated with the magnetization reversals is detected by a nearby pick-up coil as a series of voltage spikes. As the polymer swells, the distance between the triangles increases resulting in a decrease in the magnetostatic coupling, and hence both an increase in coercive force and less coherent switching characteristics, i.e. time rate of change of flux, reducing the amplitude of the voltage spike detected in the pick-up per Faraday's law (see Fig. 1b).

Applied AC and DC Fields
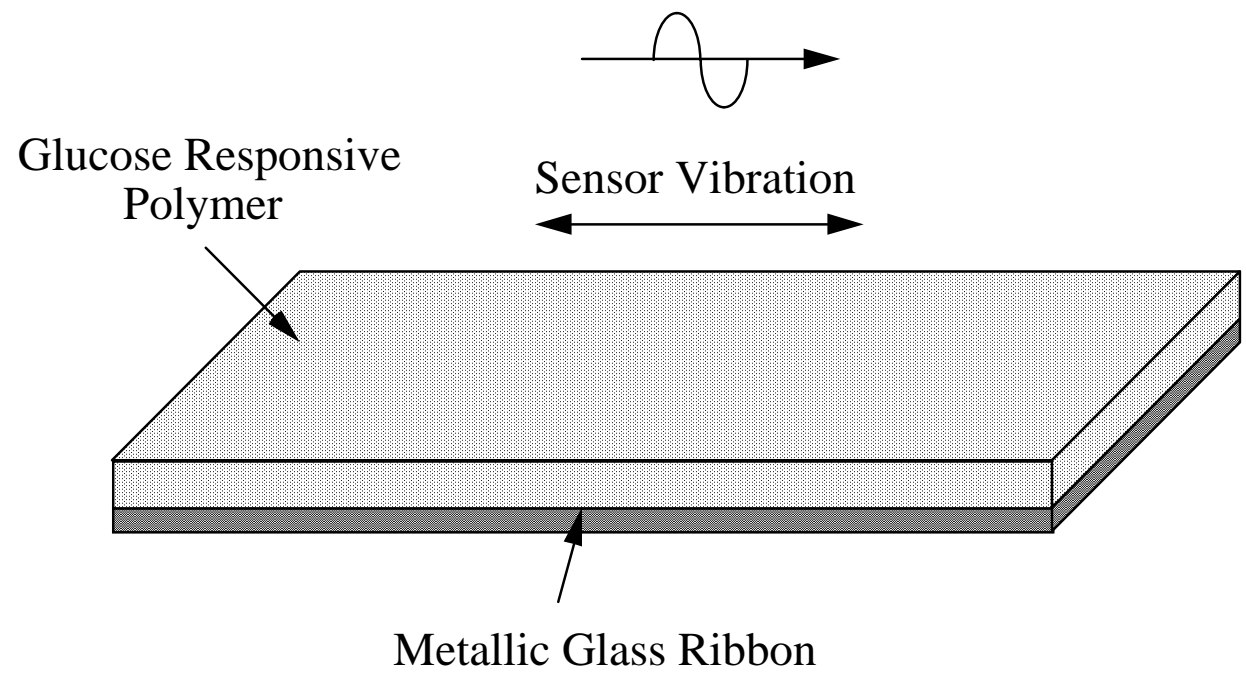

Figure 2. The magnetoelastic glucose sensor is made of an amorphous metallic glass ribbon coated with a thin layer of mass changing glucose responsive polymer.

Another magnetism-based remote query sensor design consists of a magnetoelastic Metglas ${ }^{\mathrm{TM}}$ $2626 \mathrm{MB}$ [6] ribbon that in response to an ac magnetic field demonstrates a characteristic mechanical resonant frequency. The glucose responsive polymer, described below, is adhered to the magnetoelastic ribbon as illustrated in Fig. 2. As the mass of the polymer changes in response to glucose concentration, so does the resonant frequency of the sensor. The behavior of the magnetoelastic sensor can be approximated by considering the fundamental resonant frequency $f_{0}$ of a thin rectangular plate vibrating in its basal plane [7]:

$$
f_{0}=\frac{1}{2 L} \sqrt{\frac{E}{\rho(1-\sigma)}}
$$

where $E$ is Young's modulus of elasticity, $L$ is the length of the plate, $\rho$ is the density, and $\sigma$ is the Poisson's ratio of the sensor. Provided that the mass change is small, the shift in the sensor resonant frequency with applied mass is given by:

$$
\Delta f=-f_{0} \frac{\Delta m}{2 M}
$$

where $\Delta f$ is the resonant frequency shift, $\Delta m$ is the change of mass, and $M$ is the initial mass of the sensor and the polymer layer. The negative sign in Eq. (2) indicates the resonant frequency decreases when the mass of the polymer increases. 
The polymer membrane used in this work is made of polyvinyl alcohol (PVA) and a glucose responsive co-polymer the fabrication details of which are given in the next section. In response to varying glucose concentrations the polymer changes size, absorbing corresponding amounts of solution, and thereby changing mass. The time needed for the glucose polymer to reach equilibrium depends on the membrane thickness, glucose concentration, and temperature (all measurements reported on herein were conducted at $23^{\circ} \mathrm{C}$ ).

\section{Fabrication of Glucose Responsive Polymer}

The glucose responsive polymer membrane used in our experiments was made of polyvinyl alcohol (PVA) and a co-polymer consists of N,N-Dimethylacrylamide (DMAA), butyl methacrylate (BMA), N[3-(dimethylamino) propyl] acrylamide (DMAPAA) and 3-Methacrylamido-phenylboronic acid (MAAPBA).

Preparation of raw materials: DMAA, BMA, DMAPAA, and acrylic acid were purchased from Aldrich Inc. and purified by distillation under reduced pressure before they were used. N,N'Azobisisobutyronitrile (AIBN) was obtained from Aldrich and purified by recrystallization from ethanol solution at $-10{ }^{\circ} \mathrm{C}$. MAAPBA was prepared with the following steps: $100 \mathrm{ml}$ of water and 0.1 mole of 3-aminophenylboronic acid hemisulphate were combined in a $500 \mathrm{ml}$ beaker and stirred with a magnetic stirring bar. The mixture was adjusted to $\mathrm{pH} 4.8$ with $3 \mathrm{M} \mathrm{NaOH}$ solution and cooled to $4{ }^{\circ} \mathrm{C}$. After that, 0.1 mole of 1-[3(dimethylamino) propyl]3-ethylcarbodiimide hydrochloride and 0.1 mole of acrylic acid were sequentially added while the $\mathrm{pH}$ was maintained at 4.8 and the solution temperature $4{ }^{\circ} \mathrm{C}$. After 1 hour of stirring, the mixture was extracted with diethyl ether with a reaction yield of $65 \%$.

Synthesis of glucose sensitive co-polymer: $30 \mathrm{ml}$ of ethanol, 10 mmole of MAAPBA, 10 mmole of DMAA, 10 mmole of BMA and 20 mmole of DMAPAA were combined in a $100 \mathrm{ml}$ flask. The mixture was stirred with a magnetic stirring bar and bubbled with $\mathrm{N}_{2}$ for 20 minutes. This was followed by adding $33 \mathrm{mg}$ of AIBN and then stirring and bubbling with $\mathrm{N}_{2}$ gas for another 30 minutes. The solution was heated to $70 \sim 75^{\circ} \mathrm{C}$ and the reaction was carried out for 3 hours under $\mathrm{N}_{2}$ atmosphere with stirring. The co-polymer was obtained by precipitation from diethyl ether and dried under vacuum condition. The yield was $60-75 \%$.

Formation of glucose-sensitive co-polymer and PVA films: $0.2 \mathrm{wt} \%$ glucose sensitive co-polymer solution and $0.2 \mathrm{wt} \%$ PVA solution were mixed and the mixture was cast into a model cell. After the solvents evaporated, transparent uniform films were formed. To cross-link the PVA hydroxyl groups that did not complex with the boronic acid moieties in the co-polymer, the films were immersed into $0.177 \mathrm{mmole} / \mathrm{L}$ triisocyanate pyridine solution for 10 minutes under $\mathrm{N}_{2}$ atmosphere, then the films were cross-linked in the triisocyanate pyridine solution for 4 hours at $80{ }^{\circ} \mathrm{C}$ under $\mathrm{N}_{2}$ atmosphere. The cross-linked films were dried in vacuum for 4 hours.

Glucose sensing mechanism of the polymer: When glucose complexes with the hydroxyl groups of the boronate moieties, it is inserted between the two polymer chains, increasing the distance between the chains of the co-polymer and PVA. This in turn increases the capacity of the membrane to absorb water, thereby increasing in volume and mass. When the ratio of MAAPBA in the co-polymers is increased the glucose responding PVA-membranes become harder and there is less polymer swelling. When the ratio of DMAPAA in the co-polymers is increased the glucose responding membranes 
become softer with greater swelling. It is believed that with fewer phenylboronic acid moieties, the intermolecular chains between the two polymers are weaker, so more water molecules can be absorbed, causing a higher change in volume and mass.

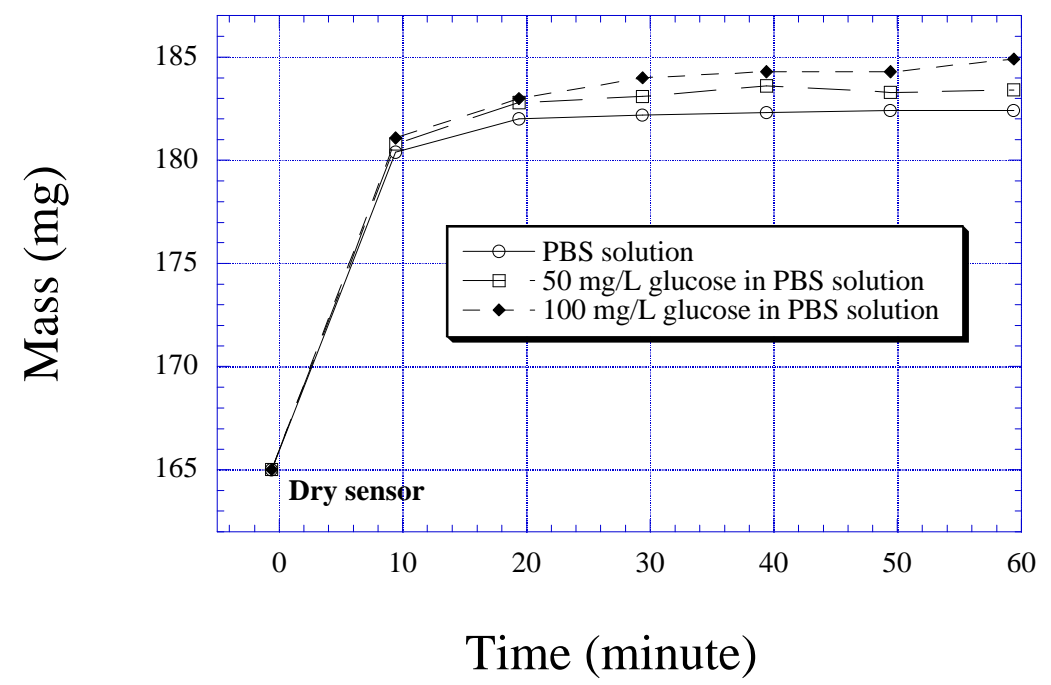

Figure 3. Mass change of a $12 \mu \mathrm{m}$ thick glucose responding polymer layer as it is immersed into phosphate buffered saline solution with and without glucose.

Fig. 3 demonstrates the change in the polymer mass as it changes from a dry to wet polymer, and as a function of glucose concentration. As seen, the largest change in mass is due to the polymer simply changing from dry to wet, however there is still a useful differential change in mass with glucose concentration. Measuring volume change is much more difficult, as the polymer softens upon immersion in solution; it was generally estimated that the volume changed approximately $5 \%$ from dry to wet.

\section{Results and Discussion}

\section{Magnetostatic Coupling Glucose Sensor}

Fig. 4 is an illustrative plot of the measured voltage spike amplitude generated by the magnetostaticbased sensor upon immersion into phosphate buffered saline (PBS) and PBS + $100 \mathrm{mg} / \mathrm{L}$ glucose. A $5 \mathrm{Oe} 10 \mathrm{~Hz}$ magnetic field was applied to the sensor through the use of a $40 \mathrm{~cm}$ diameter Helmholtz coil pair; the sensor rested within a pickup coil that consisted of a $10 \mathrm{~cm}$ diameter coil with 5,000 turns of number 40 wire. In its initial state the polymer is dry, swelling both due to immersion in water and additionally due to the glucose. It is noted that initially the amplitude of the voltage spike increases, and then decreases with time. The explanation for this is as follows: As the sensor is first immersed in the liquid the magnetic thin films upon the glucose substrate prevent rapid diffusion into the polymer from the top-side, while the liquid is free to diffuse in from the bottom. This imbalance in diffusion rates causes the sensor to initially curve upwards, forcing the magnetic elements comprising the sensor closer together thus increasing the magnetostatic coupling (coherence of magnetization change), 
resulting in a larger amplitude voltage spike upon magnetization reversal due to a greater time rate of change of flux. With time, the polymer reaches equilibrium so that the sensor once again becomes flat, regaining its initial shape. As the polymer swells with increasing glucose concentration, the effect of polymer swelling is to decrease the magnetostatic coupling between the magnetic sensor elements, reducing the coherence with which the magnetization vectors switch. Since the generated voltage amplitude is proportional to the time rate of change of flux the polymer swelling acts to reduce the measured voltage. As seen in Figure 4 the addition of glucose has resulted in additional polymer swelling, and in turn a lower measured voltage spike amplitude.

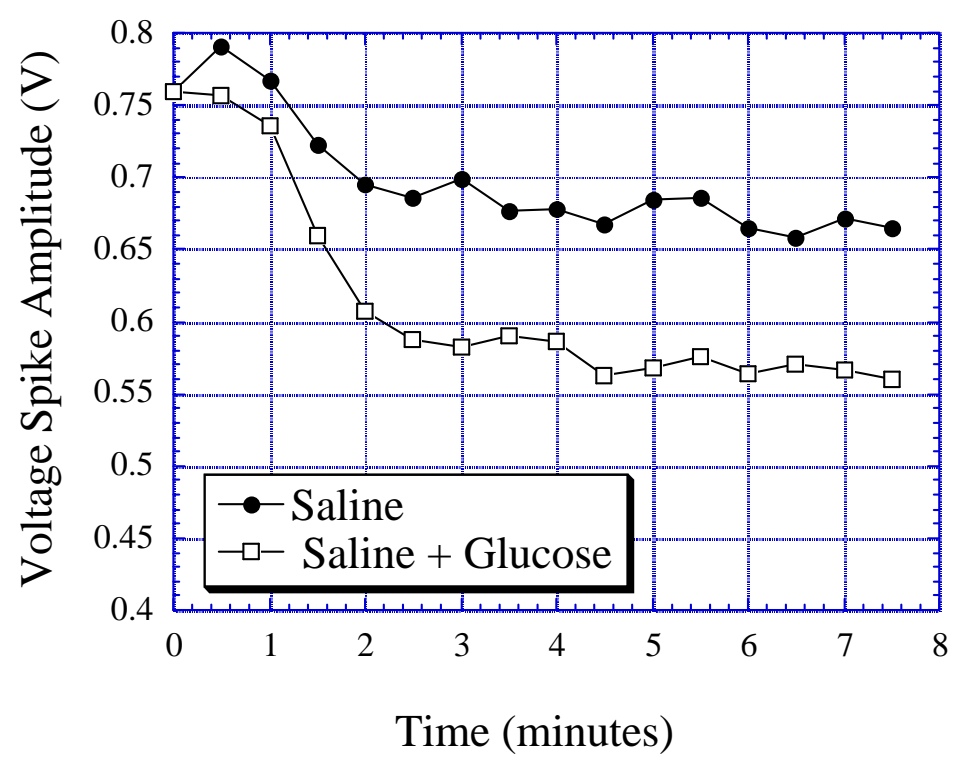

Figure 4. Measured voltage spike amplitude of a magnetostatic sensor as a function of time and glucose concentration. The sensors is placed in either a pure saline solution, or buffered saline containing $100 \mathrm{mg} / \mathrm{L}$ glucose.

There are several difficulties with the magnetostatic sensor platform worthy of discussion. One difficulty of the magnetostatic sensor is that the polymer also serves as the substrate, and as the polymer softens in solution the sensor tends to warp and buckle altering the measured signal. Furthermore, while the detected voltage spike amplitude correlates with analyte concentration, it is also a function of the relative orientation of the sensor with respect to the pickup-coil [8]. Therefore to obtain useful information from the measured amplitude of the voltage spikes a fixed distance between the pickup coil and sensor must be maintained. Independently of the voltage spike amplitude, useful information can be obtained by correlating the time-amplitude response of the sensor, which indicates the coercive force of the sensor which is dependent upon element spacing that, in turn, is dependent upon how much the glucose responsive polymer has swollen.

\section{Magnetoelastic Glucose Sensor}

Fig. 5 demonstrates the frequency spectrum of a $37.5 \mathrm{~mm} \times 12.5 \mathrm{~mm} \times 28 \mu \mathrm{m}, 2826 \mathrm{MB}$ Metglas ${ }^{\mathrm{TM}}$ $\left(\mathrm{Fe}_{40} \mathrm{Ni}_{38} \mathrm{Mo}_{4} \mathrm{~B}_{18}\right)$ [6] ribbon in air, where the resonant frequency is determined from the maximum 
amplitude of the spectrum at $58.26 \mathrm{kHz}$. The frequency of the interrogation field is swept over a predetermined frequency range and the amplitude response of the sensor measured using a lock-in amplifier connected to a $5 \mathrm{~cm}$ diameter 100 turn pickup coil. The amplitude of the interrogation field is $50 \mathrm{mOe}$, superimposed with a dc magnetic field of 5.5 Oe that serves to counterbalance the magnetic anisotropy of the sensor, optimizing both the magnetoelastic and magnetostrictive properties [9-10].

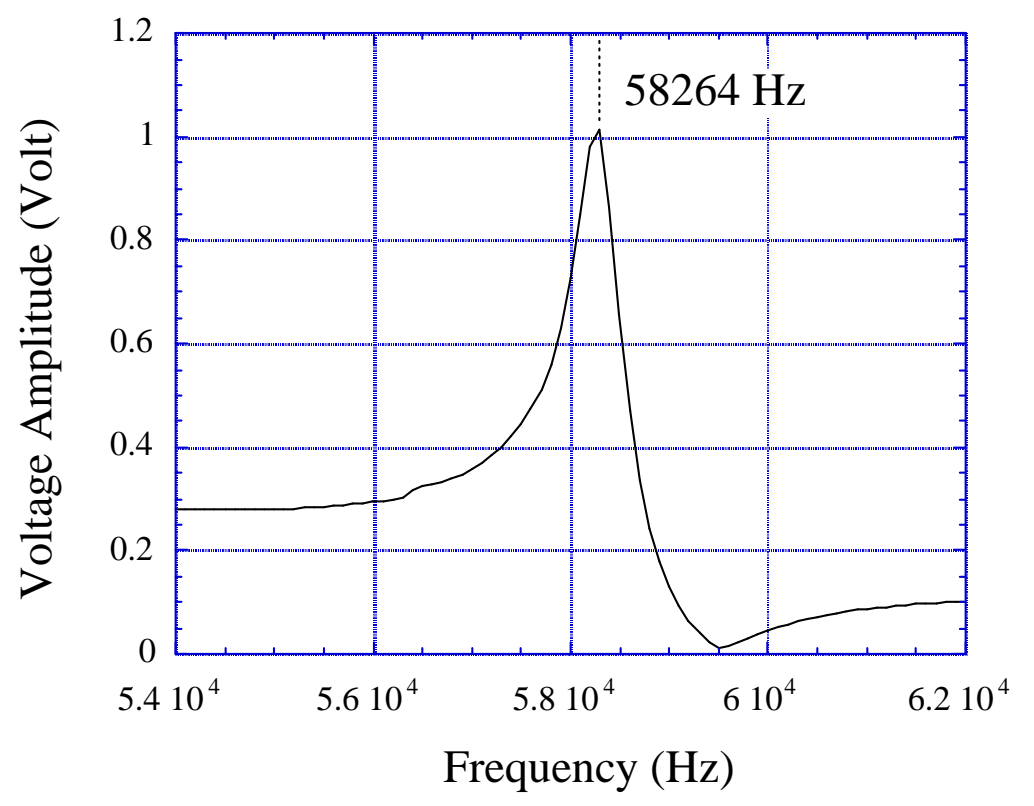

Figure 5. Illustrative frequency spectrum of a magnetoelastic ribbon in air.

The magnetoelastic glucose sensor is comprised of a Metglas ribbon coated with $4 \mu \mathrm{m}$ thick layer of the described glucose responding polymer. Fig. 6 shows the frequency spectrum of the sensor at different glucose concentrations. As shown in Fig. 6, the increase in glucose concentration, correlating to increased polymer mass, decreases the resonant frequency of the sensor and also the measured signal amplitude. This is in common with any mechanical oscillator, e.g. an acoustic bell, with mass load changing both resonant frequency and vibration amplitude. Since it is the resonant frequency that is monitored, rather than the amplitude, the response of the sensor is virtually independent of its relative orientation with respect to the pickup-coil. As dependent upon the sensitivity of the data acquisition system used, the sensors can be readily monitored over several tens of centimeters.

Fig. 7 shows the resonant frequency of the magnetoelastic sensor tracks glucose concentration linearly to $100 \mathrm{mg} / \mathrm{L}$. For each measurement, a 'wet' sensor that had already made the transition from dry polymer to wet polymer, was immersed in the solution of interest for sixty minutes prior to a measurement. The sensor was measured from low to high, and then high to low glucose concentrations without measurable drift or hysteresis. It should be noted that at high glucose concentration levels, e.g. approximately $1 \mathrm{~g} / \mathrm{L}$ and above, the effect of fluid viscosity creates a damping force on the sensor that has to be taken into account when interpreting the resonance frequency shifts [11]. 


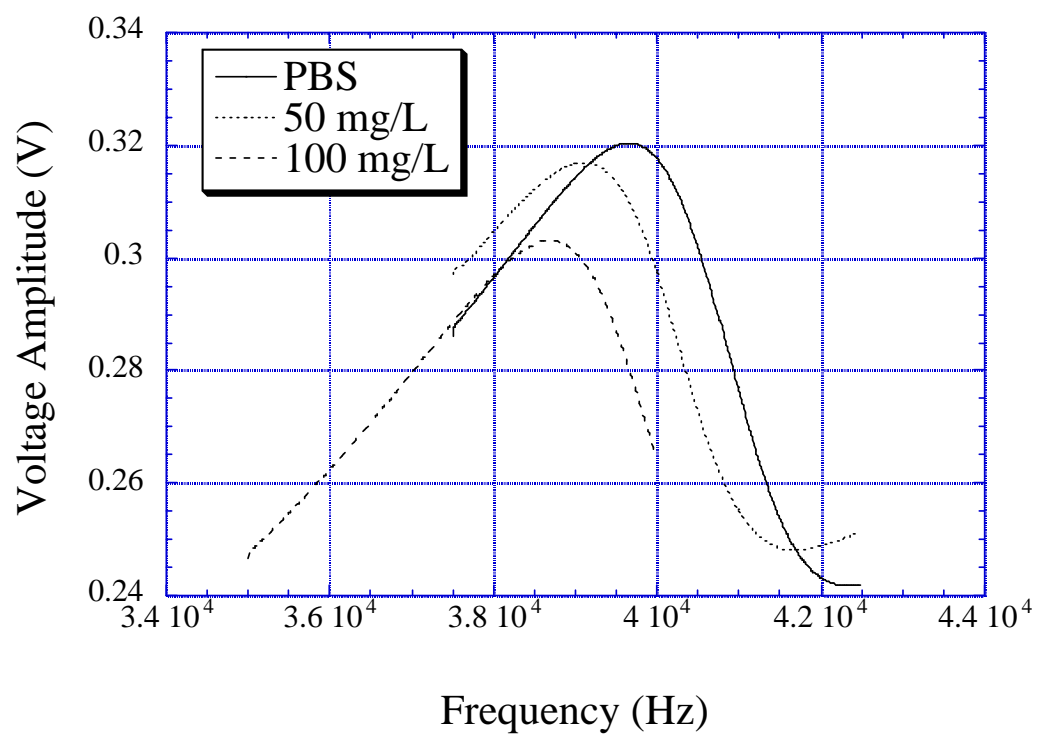

Figure 6. Frequency spectrum of a magnetoelastic glucose sensor as a function of glucose concentration.

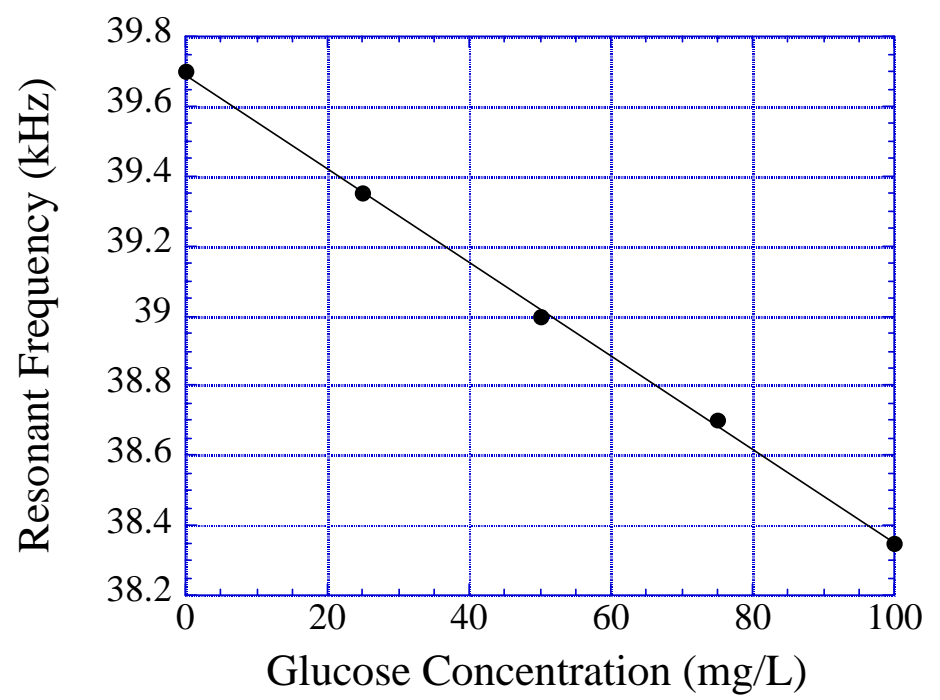

Figure 7. Shift in resonant frequency of a magnetoelastic sensor as a function of glucose concentrations. A 'wet' sensor is immersed in the glucose concentration of interest for sixty minutes to reach equilibrium prior to a measurement.

\section{Conclusions}

In combination with a mass and volume changing glucose responsive polymer, the design and application of two magnetism-based glucose sensors are presented. The magnetostatic coupling glucose sensor consists of four triangularly shaped magnetically soft thin-film elements attached to a volume-changing glucose responsive thick film polymer. As seen in Figure 3, in response to 
increasing glucose concentration the polymer swells, which also serves to increase its mass. As the polymer swells, the distance between the magnetic elements increases. This increased spacing between elements in turn results in less magnetostatic coupling, so that when the magnetization vectors of each element rotate they do so individually. The non-coherent rotation of the magnetization vectors results in a smaller time rate of change of flux and hence, as per Faraday's law, a smaller generated voltage spike amplitude in the monitoring pick-up coil. With reference to Figure 4, the initial upward trend in the amplitude of the measured voltage spike is due to curling of the sensor that brings the magnetic elements closer. After equilibrium is achieved, the glucose solution demonstrates a decrease in the detected voltage spike amplitude due to less magnetostatic coupling between elements.

Experimental results show the resonant frequency of the magnetoelastic glucose sensor linearly decreases with increasing glucose concentration due to the effect of increasing polymer mass. Although the mass of the polymer changes with glucose concentration, the saturation time for the analyte to diffuse into the homogeneous polymer layer is too long for most useful applications. Using polymer strips or beads rather than thick films could enhance the response time of the polymer.

The remote query nature of the magnetism based glucose sensors offers the possibility that, with the sensors coated with a suitable biocompatible coating such as PEG, the sensors could be placed inside a patient and monitored remotely without the need for blood sampling or direct connections. Since the sensors are passive, with no internal power supplies, they could last indefinitely as dependent upon the stability of the glucose responding polymer.

\section{Acknowledgements}

Support of this work by the National Science Foundation under grant ECS-9988598, and NASA under grant NAG-1-01036 is gratefully acknowledged.

\section{References}

1. Wu, J.; Suls, J.; Sansen, W. The glucose sensor integratable in the microchannel. Sens. Actuators B 2001, 78, 221-227.

2. $\mathrm{Xu}, \mathrm{J} . ;$ Chen, $\mathrm{H}$. Amperometric glucose sensor based on glucose oxidase immobilized in electrochemically generated poly(ethacridine). Analytica Chimica Acta, 2000, 423, 101-106.

3. Yasuzawa, M.; Matsuki, T.; Mitsui, H.; Kunugi, A.; Nakaya, T. Properties of glucose sensors prepared by the electropolymerization of pyrroles containing phosphatidylcholine (II). Sens. Actuators B 2000, 66, 25-27.

4. Mishima, Y.; Motonaka, J.; Maruyama, K.; Nakabayashi. I.; Ikeda, S. Glucose sensor based on titanium dioxide electrode modified with potassium hexacyanoferrate(III). Sens. Actuators B 2000, 65, 343-345.

5. Atalante $^{\circledR}$ film, a cobalt-based magnetically soft amorphous film made by Innovative Sputtering Technology, Zulte, Belgium.

6. Metglas ${ }^{\mathrm{TM}}$ is a trademark of Honeywell Corporation. http://www.honeywell.com.

7. Landau, L. D.; Lifshitz, E. M. Theory of Elasticity, 3rd. ed., Pergamon, 1986; Ch.II. 
8. Grimes, C. A.; Seitz, W. R.; Horn, J.; Doherty, S. A.; Rooney, M. T. A remotely interrogatable magnetochemical pH sensor. IEEE Trans. Magnetics 1997, 33, 3412-3414.

9. Livingston, J. D. Magnetomechanical properties of amorphous metals. Phys. Stat. Sol. A 1982, 591-596.

10. Garcia-Arribas, A.; Barandiaran, J. M.; Gutierrez, J.; Sagastabeitia, I. On the origin of the nonlinear and chaotic behavior of the magnetoelastic resonance. J. Appl. Phys. 1997, 81(9), 56865688.

11. Stoyanov, P. G.; Grimes, C. A. A remote query magnetostrictive viscosity sensor. Sens. Actuators 2000, 80, 8-14.

Sample Availability: Available from the author.

(C) 2001 by MDPI (http://www.mdpi.net). Reproduction is permitted for noncommercial purposes. 